\title{
OCR Based Image Equation Calculator
}

\author{
Tripti Goyal ${ }^{1}$, Vipul Goyal ${ }^{2}$ \\ M.Tech Student, Electronics Department, Bhagwant University, Ajmer, India ${ }^{1}$ \\ MS Student, Electrical and Computer Engineering Department, University of Texas, Austin, US ${ }^{2}$
}

\begin{abstract}
This paper introduces the design and implementation of an OCR based equation solver in MATLAB which can recognize the formula captured by a mobile phone camera and then computes and displays the result in MATLAB. This application enables a faster calculation by avoiding inputting the formula to some device. In this paper we demonstrate equation solver that takes a camera image of an equation and displays the solution. The papers we referred to were capable of solving simple arithmetic equations (addition, subtraction, multiplication and division). We modified the approach and are able to solve systems of linear equations of up to three variables.
\end{abstract}

Keywords: Binarization, Bounding Box, Rotation, OCR, Calculator.

\section{INTRODUCTION}

Depending on the assistive device, the equations need to been teredina specific format. Our goal is to develop an application that bridges the gap between technology and the traditional pen and paper approach in an intuitive manner. The user can capture a camera image of a mathematical expression and just see the result.

\section{CAPTURING IMAGE}

Here we will capture an image of an equation using any good resolution camera.

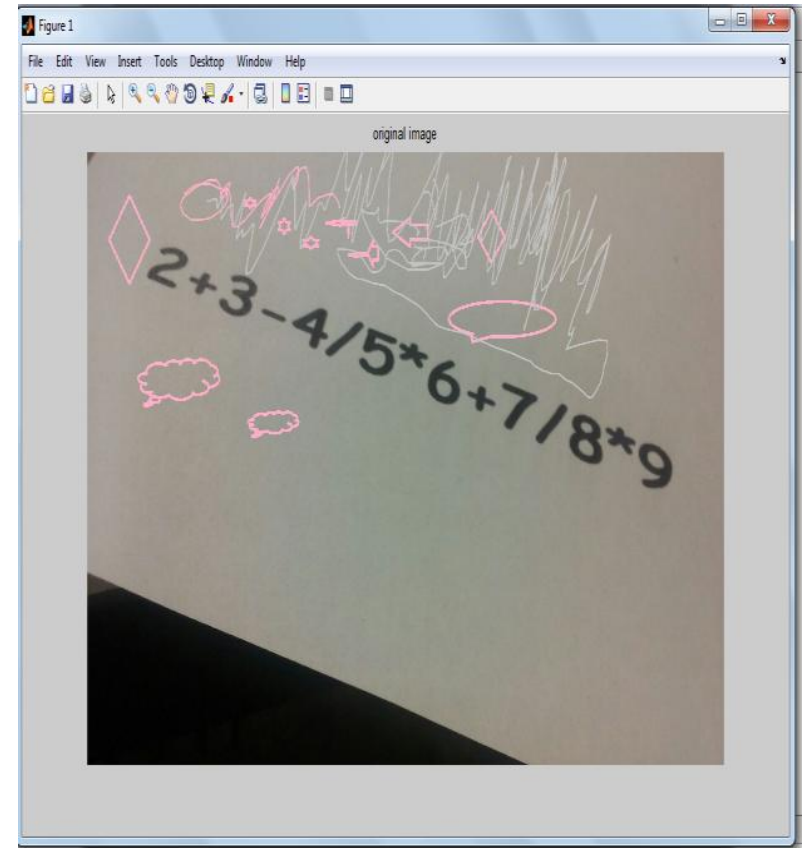

Figure 1: Original Image

\section{BINARIZATION}

The image is downsized to $640 * 480$ pixels from its original size to speed up transmission to server. The resulted image is the background of the original image. By subtracting it from original image, we get a uniformly lighted image which is safe to undergo global thresholding.

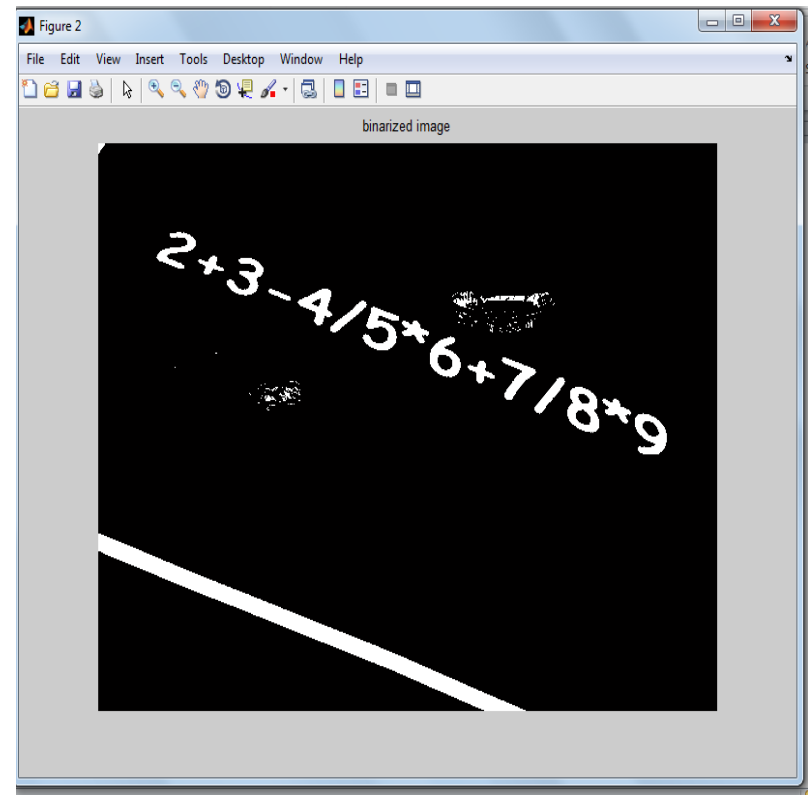

Figure 2:-Binarized Image

\section{LOCALIZATION}

The localization of the equation in the image is done in three phases:

A. Remove extremely small regions

We define these extremely small regions as having area of only order of 10 pixels, because these regions cannot be part of the formula.

B. Get rid of regions having area of small amount of counts in the image

We are assuming regions belonging to the formula should have similar areas which occur at least three times. Because we do not know the area of formula (area changes depending on zoom in and out of targets when the picture is taken), we have to find statistics of the areas of regions and process the image accordingly. This process is only done if the relative difference between maximum and minimum area is larger than 10 (if the image only contain target then there is no need to filter according to area), otherwise we skip this step. 
C: Separate image into patches

After filtering the image according to area property, the regions left should not contain clutters having unusual area. Normally what has been removed are chunk region in the background. We then need to find patches in the image that can potentially be out target. Usually these include formula and other text clutter in the image. To separate them, we're assuming that the texts in the same patch should have the same size and in order to separate one patch from another, texts in different patch should have different size. This assumption is reasonable in the sense that most of the time clutters are of different size from target, otherwise there is no way to distinguish one from another.

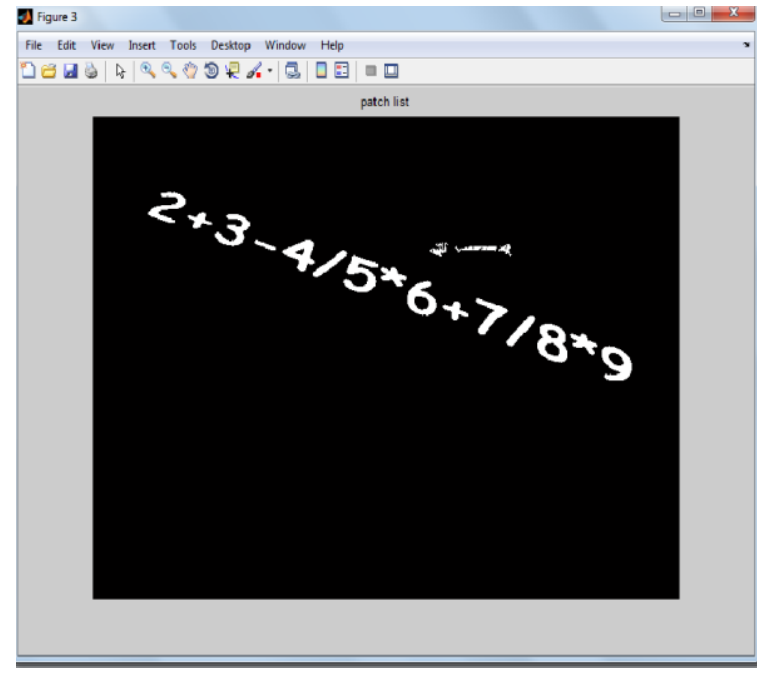

Figure 3: patched imaged

\section{BOUNDING BOX}

Regions in the binary image is labelled and their centroid and bounding box is calculated

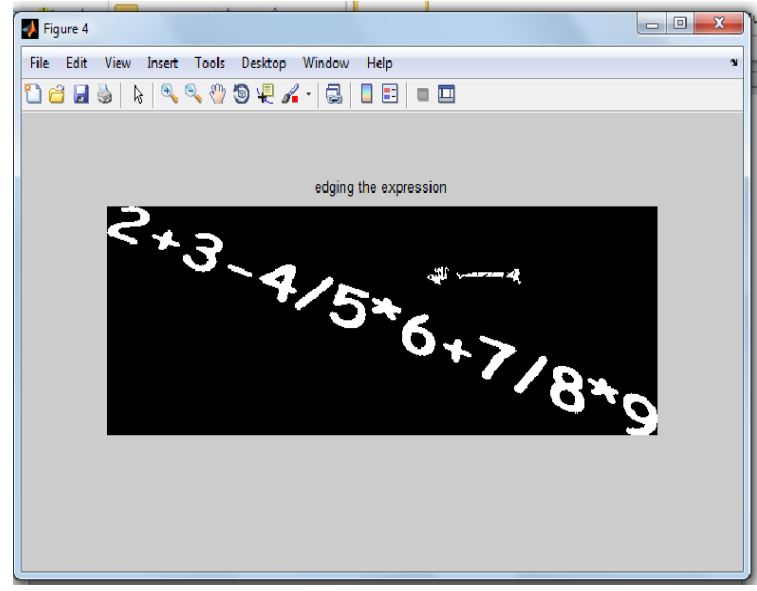

Figure 4 edged expression

\section{ROTATION}

The images taken are highly possible to be rotated or have some perspective distortions. This will lower the recognition ability of OCR. We did an adjustment on the rotated or oblique images before feeding each detected candidates into OCR.

\section{A. Rotation Adjustment:}

Considering the formula might not be in a horizontal line, rotation is needed. First label all the regions of the image, and find their centroids. If the y values (the value in the vertical direction) of the first label and the last label are very similar (the difference is less than 2 ), rotation is not needed, because the formula is almost horizontal. If the difference is bigger than that, calculate the horizontal difference and vertical difference of the first and last label, and then calculate the corresponding angle, and finally rotate the image to horizontal level.

\section{B.Perceptive Adjustment:}

We use the method of perspective transformation to adjust the oblique image.Users are highly likely to take pictures by an oblique angle, other cases merely happen. Hence, our simplification is reasonable.

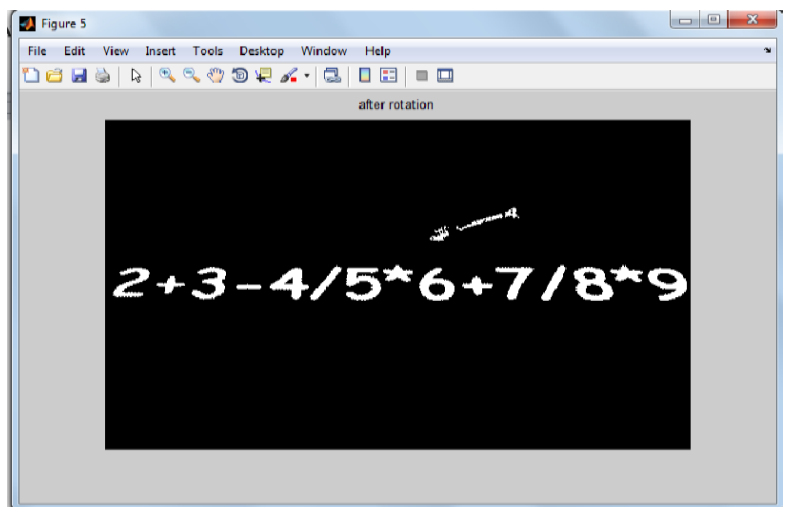

Figure 5 rotating the oblique image

\section{OCR}

In order to improve detection accuracy the number of characters in the database are limited to digits (0-9), two variables $(\mathrm{a}, \mathrm{b})$, and a subset of mathematical operators $(+$, ,$- \mathrm{x}$, and=). These characters are sufficient for composing both quadratic equations and the majority of a system of linear equations in two variables. The dictionary can be easily expanded to include more characters in the future.

The accuracy of optical text- recognition algorithms improves significantly if perspective distortion is first corrected. Following a similar method, the top line and baseline of the regions in the segmented expression are found.

\section{MODIFICATION}

When reference was made from some research papers [5] we found solutions of single variable equations but here we have introduced the solution for three variable equations.

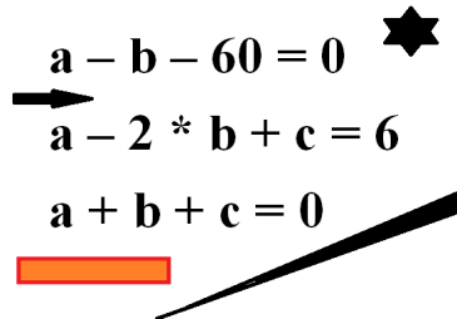

Figure 6: Input image 
INTERNATIONAL JOURNAL OF INNOVATIVE RESEARCH IN ELECTRICAL, ELECTRONICS, INSTRUMENTATION AND CONTROL ENGINEERING Vol. 4, Issue 3, March 2016

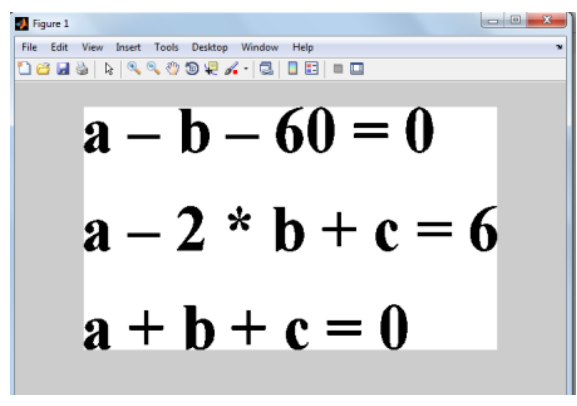

Figure 7: Image after processing

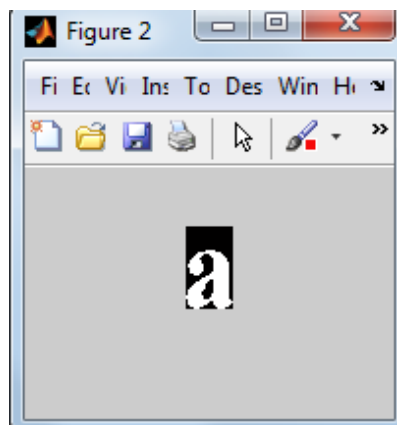

Figure 8: I element detected

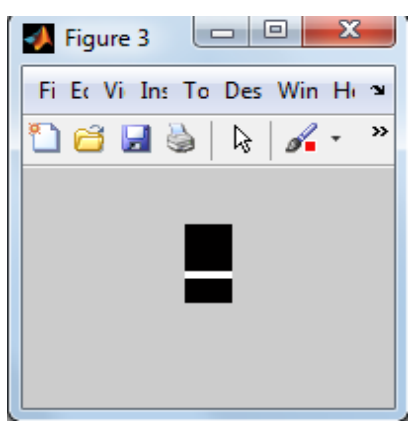

Figure 9: II element detected

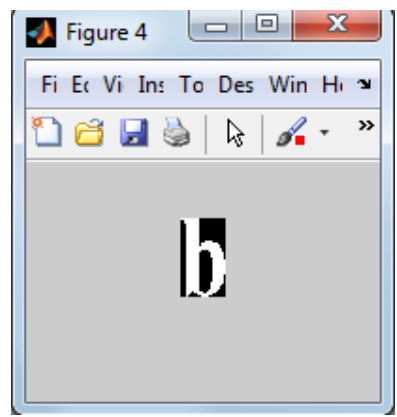

Figure 10: III element detected

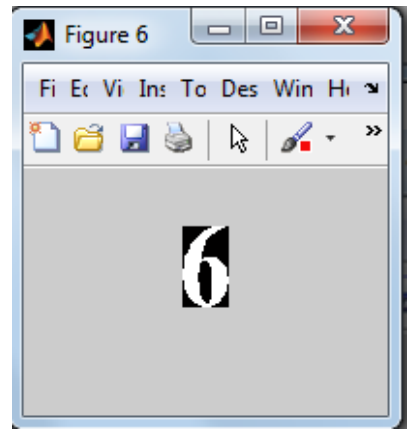

Figure 11: IV element detected

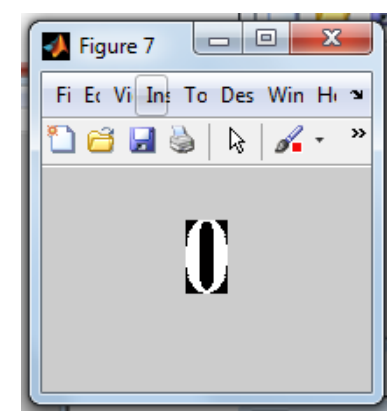

Figure 12: V element detected

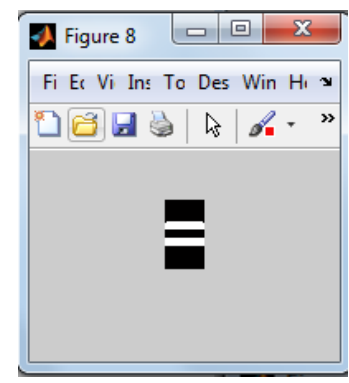

Figure 13: VI element detected

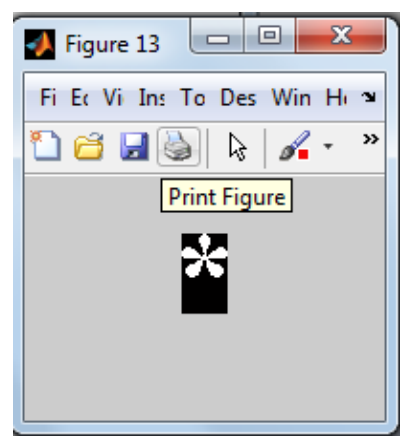

Figure 14: VII element detected

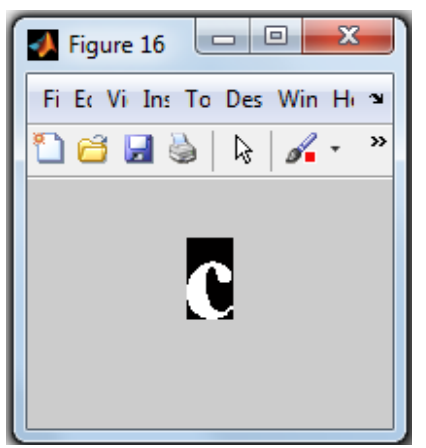

Figure 15: VIII element detected

\section{RESULT}

The result which is obtained on CONSOLE can be summarized as:-

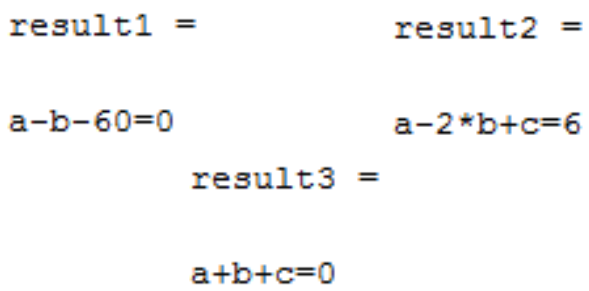




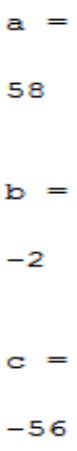

Figure 16: console output

\section{CONCLUSION}

The equation solving process is made quite easier without the need of pen paper mechanism. The image which is clicked at high resolution is first brought down to processing level and then unwanted parts are removed ,equation is bounded by a algorithm, this further removes redundant pixel .Finally the image is sent to OCR where each letter is detected and then finally result is shown.

\section{REFERENCES}

[1] VLFEAT Open Source Library. Available: http://www. vlfeat.org/

[2] Tesseract Optical character Recognization engine. Available: http://code.google.com/p/tesseract-ocr/

[3] Sam Tsai, Huizhong Chen, David Chen, Ramakrishna Vedantham, Radek Grzeszczuk, and Bernd Girod, "Mobile Visual Search UsingI mage and Text Features", Proc. $45^{\text {th }}$ Annual Asilomar Conference on Signals, Systems and Computers, November 2011.

[4] www.mathworks.com/help/toolbox/images/ref/ imtransform.html

[5] Mobile Camera-based Calculator code by Jingyi Dai, Li wei Wang: http://web.stanford.edu/ class/ee368/Project_12/index.html

\section{BIOGRAPHY}

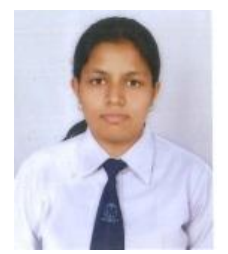

Tripti Goyal received her B.Tech in ECE from Govt. Women Engg College Ajmer, India and currently pursuing M.Tech from Bhagwant University India.

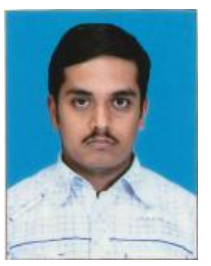

Vipul Goyal received his B.Tech degree in Electrical Engineering from IIT Gandhinagar in 2013. He then worked as a Design Engineer at Texas Instruments Bangalore for two years and is currently enrolled in MS programme in Electrical and Computer Eng. at The University of Texas at Austin. 